\title{
Genomic aberrations of the CACNA2D1 gene in three patients with epilepsy and intellectual disability
}

\author{
Sarah Vergult ${ }^{1}$, Annelies Dheedene ${ }^{1}$, Alfred Meurs ${ }^{2}$, Fran Faes ${ }^{3}$, Bertrand Isidor ${ }^{4}$, Sandra Janssens ${ }^{1}$, \\ Agnès Gautier ${ }^{5}$, Cédric Le Caignec ${ }^{4}$ and Björn Menten ${ }^{\star, 1}$
}

\begin{abstract}
Voltage-gated calcium channels have an important role in neurotransmission. Aberrations affecting genes encoding the alpha subunit of these channels have been associated with epilepsy and neuropsychiatric disorders such as autism or schizophrenia. Here we report three patients with a genomic aberration affecting the CACNA2D1 gene encoding the $\alpha_{2} \delta$ subunit of these voltage-gated calcium channels. All three patients present with epilepsy and intellectual disability pinpointing the CACNA2D1 gene as an interesting candidate gene for these clinical features. Besides these characteristics, patient 2 also presents with obesity with hyperinsulinism, which is very likely to be caused by deletion of the CD36 gene.
\end{abstract}

European Journal of Human Genetics (2015) 23, 628-632; doi:10.1038/ejhg.2014.141; published online 30 July 2014

\section{INTRODUCTION}

An essential step in neurotransmission is the influx of $\mathrm{Ca}^{2+}$ through voltage-gated calcium channels. These voltage-dependent calcium channels have been described to be associated with epilepsy and several neuropsychiatric disorders such as autism and schizophrenia. ${ }^{1-3}$ Typically, these channels are composed of four subunits: an alpha1, alpha2-delta, beta and gamma subunit, each encoded by several genes. The $\alpha_{1}$ subunit is the primary subunit necessary for channel functioning and in total 10 genes are known to encode this subunit. The $\alpha_{2} \delta$ subunit consists of two peptides, the alpha2 and the delta peptide. They are posttranslationally cleaved and subsequently linked by disulfide bonds. Four genes are known to encode these subunits (CACNA2D1, CACNA2D2, CACNA2D3 and CACNA2D4). ${ }^{4}$ Co-expression of the $\alpha_{2} \delta$ genes enhances the level of expression of the $\alpha_{1}$ subunit and causes an increase in current amplitude. The beta subunit has a major role in stabilizing the final $\alpha_{1}$ subunit conformation and delivering it to the cell membrane. ${ }^{4}$

Interestingly, the $\alpha_{2} \delta$ subunits encoded by CACNA2D1 and CACNA2D2 are targets for gabapentinoid drugs, pregabalin and gabapentin, two anti-epileptic drugs. ${ }^{5}$ Mice bearing point mutations in the CACNA2D1 gene have a decreased response to pregabalin as well as an abnormal central nervous system synaptic transmission. ${ }^{6}$ Furthermore, the $\alpha_{2} \delta$ subunit encoded by the CACNA2D1 gene is widely expressed in the nervous system, especially in neuronal cell types. ${ }^{7}$ This suggests a potential role for CACNA2D1 in the pathogenesis of epilepsy.

Here we report on three patients with epilepsy and intellectual disability (ID) with a genomic aberration affecting the CACNA2D1 gene, pinpointing this gene as an interesting candidate gene for epilepsy and ID.

\section{MATERIALS AND METHODS \\ Clinical reports}

Patient 1 (Belgium). Patient 1 is the fifth child of healthy non-consanguineous parents. Family history is negative for epilepsy or other developmental problems. She was born at term in breech position after an uneventful pregnancy. Birth weight $(3 \mathrm{~kg})$, height $(48 \mathrm{~cm})$ and head circumference were normal. At the age of 1 year, a global developmental delay was diagnosed and physiotherapy was started. The first epileptic seizures occurred at the age of 3 years and were characterized by twitching of the face and the left arm. She also had generalized tonic-clonic seizures with loss of consciousness and urine loss. EEGs at that time showed multifocal spikes and spike complexes mainly in the right hemisphere. Later she also developed shocks and atonic seizures. Although several drugs were tested, daily epileptic seizures persisted. MRI of the brain revealed bilateral frontotemporal polymicrogyria, mega cisterna magna and a cyst in the cavum veli interpositi, under the splenium of the corpus callosum expanding posterior to the supravermian cistern.

At the age of 15 years, she is not able to speak in sentences and has difficulties walking long distances. IQ testing revealed an overall IQ around 50. Upon clinical examination at this age, her height is $150 \mathrm{~cm}(<\mathrm{P} 5)$, weight $57 \mathrm{~kg}$ (P75) and head circumference $54.5 \mathrm{~cm}$ (P50). Besides the psychomotor difficulties, she still suffers from epilepsy for which she is now treated with topiramate, valproic acid, lamotrigine, oxcarbazepine and clobazam. The last EEG was abnormal with multifocal epileptic activity.

Patient 2 (Belgium, DECIPHER patient 285588). Patient 2 is the second child of healthy non-consanguineous parents. She was born at term after an uneventful pregnancy. Birth weight $(3,8 \mathrm{~kg})$, length $(50 \mathrm{~cm})$ and head circumference $(33,5 \mathrm{~cm})$ were normal. Initial neuromotor development appeared near normal with independent sitting at the age of 9 months and walking at 18 months. At the age of 15 months, she experienced a first generalized tonic-clonic seizure during a febrile episode. MRI of the brain was normal, EEG showed slowing over the left parasaggital leads but no epileptic abnormalities. Despite treatment with valproic acid, she continued to

${ }^{1}$ Center for Medical Genetics, Ghent University, Ghent University Hospital, Ghent, Belgium; ${ }^{2}$ Laboratory for Clinical and Experimental Neurophysiology, Neurobiology and Neuropsychology, Department of Neurology, Ghent University, Ghent University Hospital, Ghent, Belgium; ${ }^{3}$ Centrum voor Ontwikkelingsstoornissen (COS), Ghent University Hospital, Ghent, Belgium; ${ }^{4}$ Service de Génétique Médicale, Institut de Biologie, Nantes, France; ${ }^{5}$ Service de Pédiatrie, CHU, Nantes, France

${ }^{*}$ Correspondence: Dr B Menten, Center for Medical Genetics, Ghent University, Ghent University Hospital, De Pintelaan 185, Gent 9000, Belgium. Tel: + 329 3325284 Fax: + 329 3326549; E-mail: Bjorn.Menten@Ugent.be

Received 4 December 2013; revised 13 June 2014; accepted 25 June 2014; published online 30 July 2014 
experience seizures between the ages of 21 months and 6 years. These seizures were characterized by generalized tonic contraction of limbs and trunk, revulsion of the eyes and loss of consciousness, and sometimes necessitated intubation. After one such seizure, a transient postictal paresis of the right arm and leg was observed, suggesting the possibility of a focal onset in the left hemisphere. MRI at the age of 3 years showed global cortical atrophy. From the age of 7 years, she became seizure-free under valproic acid treatment. After the first epileptic seizure, a delayed psychomotor development was noticed. Every subsequent seizure also resulted in a slight regression in psychomotor development. In addition, gait ataxia was noted on clinical exam from the age of 36 months. Her IQ is estimated around 80 and she followed special education. Clinical examination shows some craniofacial dysmorphic features with hypertelorism and striking blue irises. Inspection of the skin shows multiple ephelides spread throughout the body, mostly pronounced in the face, neck and upper limbs. There are no café-au-lait spots nor cutaneous neurofibromes. There is lymphedema of the left foot, as a result of a surgical procedure at the age of 10 years for lymphangitis. She is still relatively obese $(\mathrm{BMI}=25$, among healthy diet) and has a short stature (length P10). Head circumference is at the 75th percentile. From 11 to 15 years she has had transient diabetes with hyperinsulinemia, which disappeared under diet.

Patient 3 (France, DECIPHER patient 259199). Patient 3 was born at term to non-consanguineous parents. Her developmental milestones were significantly delayed; speech delay was noticed and she was only able to walk at the age of 3 years. Although she follows special education, at the age of 16 years, she is still not able to read or write. Generalized epileptic seizures first occurred at the age of 5 years. The EEG showed frontotemporal spikes, brain MRI did not reveal any abnormalities. Treatment with lamotrigine was initiated, and valproic acid was subsequently associated. Under this treatment, the EEG normalized but she continued to suffer from rare epileptic seizures (one/year).

Clinical examination at the age of 16 years reveals facial dysmorphism with synophris, short nose, open mouth, everted lower lip, thick lips and low set ears. Clinodactyly V, brachymetacarpy and -tarsy $\mathrm{V}$ are also observed. Weight $(55.8 \mathrm{~kg})$, height $(1.66 \mathrm{~m})$ and head circumference $(54 \mathrm{~cm})$ are normal at this age.

\section{Cytogenetic analyses}

Analysis of G-banded metaphase chromosomes from patient 1 and her parents was performed on short-term lymphocyte cultures using standard procedures. Fluorescence in situ hybridization (FISH) was performed as previously described. ${ }^{8}$ FISH probes were selected from the $1 \mathrm{Mb}$ and $32 \mathrm{~K}$ set clones via the Ensembl Genome Browser website (http://www.ensembl.org/). Table 1 shows the FISH probes that were used in the FISH experiments.

\section{Array CGH}

DNA was isolated from total blood using the QiaAmp Purification Kit (Qiagen, Venlo, The Netherlands) according to the manufacturer's instructions. DNA was hybridized on the Agilent Human Genome CGH Microarray $180 \mathrm{~K}$ (AMADID\#014950, Agilent Technologies, Diegem, Belgium), according to the manufacturer's instructions with minor modifications. ${ }^{9}$

Array CGH data were analyzed with arrayCGHbase ${ }^{10}$ for copy number analysis.

\section{$\mathrm{X}$-inactivation assay}

Examination of the methylation pattern at the androgen receptor $(A R)$ locus was performed on genomic DNA isolated from blood leucocytes according to Allen et al. ${ }^{11}$

\section{RNA isolation}

Epstein-Barr virus-transformed B-lymphocyte cell lines were derived from patient 1 and five age- and sex-matched controls. These cell lines were grown in standard conditions. RNA was isolated using the miRNeasy kit (Qiagen, Venlo, the Netherlands) according to the manufacturer's instructions.
Table 1 FISH probes used to map the translocation breakpoints in patient 1

\begin{tabular}{|c|c|c|c|c|}
\hline Name & Chromosome & Start (bp) & Stop (bp) & Band \\
\hline RP11-445016 & $x$ & 56961277 & 57116324 & Xp11.21 \\
\hline RP5-966K21 & $x$ & 57800610 & 57942294 & Xp11.21 \\
\hline CEP $X$ & $x$ & \multicolumn{2}{|c|}{ Alpha satelites } & Xp11.1-q11.1 \\
\hline RP13-34C21 & $x$ & 62352481 & 62545017 & Xq11.1 \\
\hline RP4-80804 & $x$ & 66661968 & 66798883 & Xq12 \\
\hline RP11-523P2 & $x$ & 71453357 & 71646277 & Xq13.1 \\
\hline RP11-236012 & $x$ & 74785655 & 74948375 & Xq13.3 \\
\hline RP11-467H10 & 7 & 76653766 & 76848310 & $7 q 11.23$ \\
\hline RP11-28I21 & 7 & 81069104 & 81244871 & $7 q 21.11$ \\
\hline RP4-560014 & 7 & 81519860 & 81662483 & $7 q 21.11$ \\
\hline RP4-802A9 & 7 & 81805236 & 81871308 & $7 q 21.11$ \\
\hline RP11-556B13 & 7 & 81880897 & 82024986 & $7 q 21.11$ \\
\hline RP11-560N24 & 7 & 81983189 & 82156396 & $7 q 21.11$ \\
\hline RP4-808G16 & 7 & 82100774 & 82265084 & $7 q 21.11$ \\
\hline RP4-740B21 & 7 & 82265085 & 82384337 & $7 q 21.11$ \\
\hline RP11-66504 & 7 & 82870949 & 83041443 & $7 q 21.11$ \\
\hline RP4-649P17 & 7 & 83685666 & 83820679 & 7q21.11 \\
\hline RP11-727N2 & 7 & 84482187 & 84592771 & $7 q 21.11$ \\
\hline RP11-533L22 & 7 & 84892747 & 85065530 & $7 q 21.11$ \\
\hline RP11-212B1 & 7 & 87069276 & 87215419 & $7 q 21.12$ \\
\hline RP11-263N12 & 7 & 89484415 & 89635180 & $7 q 21.13$ \\
\hline RP5-1099C19 & 7 & 92136269 & 92251541 & $7 q 21.2$ \\
\hline RP11-95A10 & 7 & 95121078 & 95310527 & $7 q 21.3$ \\
\hline RP11-333G13 & 7 & 101389774 & 101602962 & 7q22.1 \\
\hline RP11-22N19 & 7 & 105797662 & 105975160 & $7 q 22.2$ \\
\hline RP11-5N18 & 7 & 108130860 & 108318995 & $7 q 31.1$ \\
\hline
\end{tabular}

Abbreviation: FISH, fluorescence in situ hybridization.Probe name, chromosome, start and end positions according to the GRCh37 genome assembly and chromosome band are given for each probe. The probes encompassing or flanking the translocation breakpoints are in bold.

\section{Quantitative PCR}

cDNA synthesis was performed on $2 \mu \mathrm{g}$ total RNA with $4 \mu \mathrm{l}$ of iScript reaction mix and $1 \mu \mathrm{l}$ of iScript reverse transcriptase (iScript, Bio-Rad, Nazareth, Belgium) in a final volume of $20 \mu \mathrm{l}$. This mix was then incubated for $5^{\prime}$ at $25^{\circ} \mathrm{C}, 30^{\prime}$ at $42^{\circ} \mathrm{C}$ and $5^{\prime}$ at $85^{\circ} \mathrm{C}$ using the iCycler (Bio-Rad). qPCR was performed with $5 \mathrm{ng} \mathrm{cDNA}, 2.5 \mu \mathrm{l}$ of ssoAdvanced mastermix (Bio-Rad) and $5 \mu \mathrm{m}$ primers in a total volume of $5 \mu \mathrm{l}$. Normalization of expression levels was done against three reference genes TBP, UBC and HPRT1. Analysis was performed using the qBasePlus software (http://www.biogazelle.com). Primer sequences are available on request.

\section{RESULTS}

\section{Patient 1}

Conventional karyotyping revealed a de novo apparently balanced reciprocal translocation $46, \mathrm{X}, \mathrm{t}(\mathrm{X} ; 7)(\mathrm{p} 10 ; \mathrm{q} 21.2)$ in patient 1 (Figure 1a). The translocation breakpoints were further mapped using FISH. BAC-probes RP11-556B13 and RP11-560N24 directly flank the breakpoint on chromosome 7 (Figure 1b). On the $\mathrm{X}$-chromosome, the breakpoint resides in the centromeric region, demonstrated by the split signal of the centromeric probe (Figure 1c), whereas on chromosome 7 the CACNA2D1 gene is disrupted (Figure 2). Molecular karyotyping did not reveal any aberrations near the breakpoint regions for patient 1 or any other causal CNVs.

The X-inactivation assay revealed a total (100:0) skewing of the $\mathrm{X}$-chromosome inactivation.

Expression analysis of the ARHGEF9 gene, located close to the breakpoint on chromosome $\mathrm{X}$, was performed in patient 1 and five sex- and age-matched controls. No significant difference was observed 

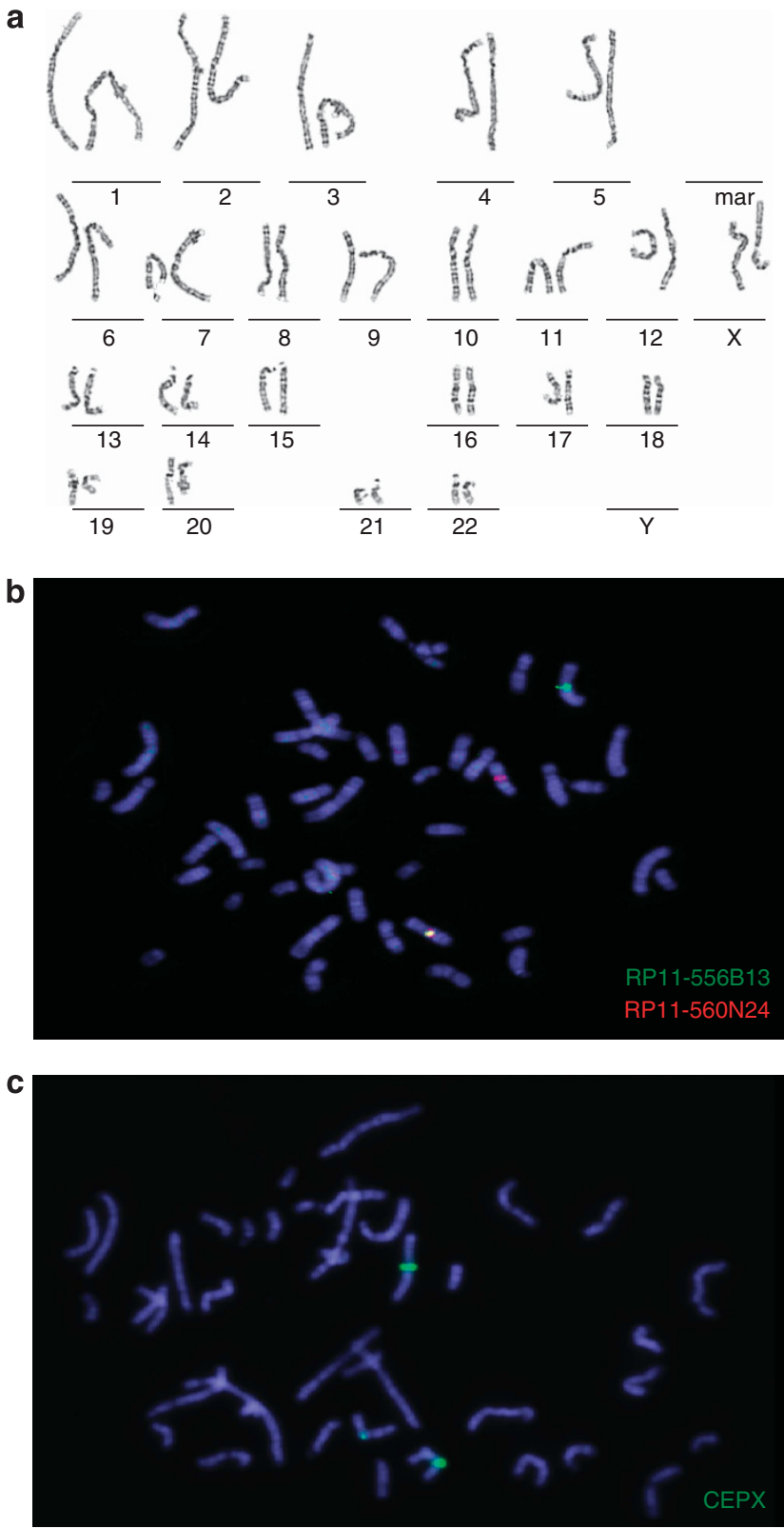

Figure 1 (a) Karyogram of patient 1. (b) RP11-556B13 (green signal) and RP11-560N24 (red signal) flanking the translocation breakpoint on chromosome 7. (c) Disruption of the X-chromosome centromere as shown by the interrupted centromeric probe. in expression in patient 1 in comparison with these controls (Supplementary Figure 1).

\section{Patient 2}

Array CGH analysis revealed a de novo $7.5 \mathrm{Mb}$ deletion chr7.hg19:g. (79,314,673_79,337,679)_(86,790,392_86,817,601)del dn (ISCN: arr[hg19] 7q21.11q21.12(79,337,679-86,790,392)x1 dn) on chromosome band 7q21.11q21.12 harboring 14 genes (Figure 2) and a maternally inherited $150 \mathrm{~kb}$ deletion on chromosome band 16q24.1.

\section{Patient 3}

A $2.72 \mathrm{Mb}$ deletion on chromosome band 7q21.11 chr7.hg19:g $\left(81,161,088 \_81,217,070\right) \_\left(83,933,784 \_84,014,514\right)$ del mat (ISCN: arr[hg19] 7q21.11q21.11(81,217,070-83,933,784)x1 mat) was detected in patient 3 containing five genes (Figure 2). This deletion was inherited from the mother who presents with mild ID and speech delay. She is also illiterate.

The data of all three patients were submitted to ClinVar (http:// www.ncbi.nlm.nih.gov/clinvar/, accession numbers SCV000165997SCV000165999).

\section{DISCUSSION}

We describe here three patients with epilepsy and ID. In patient 1 , the $\mathrm{t}(\mathrm{X} ; 7)$ translocation breakpoint on chromosome 7 disrupts the CACNA2D1 gene. In patient 2 and 3, the smallest region of overlap shared by the deletions contains only five genes (HGF, CACNA2D1, $P C L O, S E M A 3 E, S E M A 3 A)$, including the CACNA2D1 gene. These data suggest that disruption or deletion of CACNA2D1 is very likely to be associated with the epilepsy observed in these patients.

In patient 1 , the epilepsy is associated with polymicrogyria, which is not observed in patients 2 and 3, or was this noted in two patients with overlapping deletions previously reported by Mefford et al. ${ }^{12}$ These two patients, respectively, present with myoclonic astatic epilepsy and symptomatic generalized epilepsy. All three patients reported here suffer from generalized epileptic seizures. In patient 1 focal motor, generalized tonic-clonic, atonic and myoclonic seizures were observed, suggesting multifocal or symptomatic generalized epilepsy. Patient 2 presents with generalized tonic(-clonic) seizures. In this patient, there is limited evidence for focal onset of the seizures. The age at seizure onset ranged from 21 months in patient 2 to 3 and 5 years of age in patient 1 and 3. In the patients from Mefford et al, seizure onset was observed at 2 months of age (patient T438) and 9 months (patient T964).

In patient T438 with myoclonic astatic epilepsy, the deletion segregated in the family with a spectrum of epilepsy and cognitive

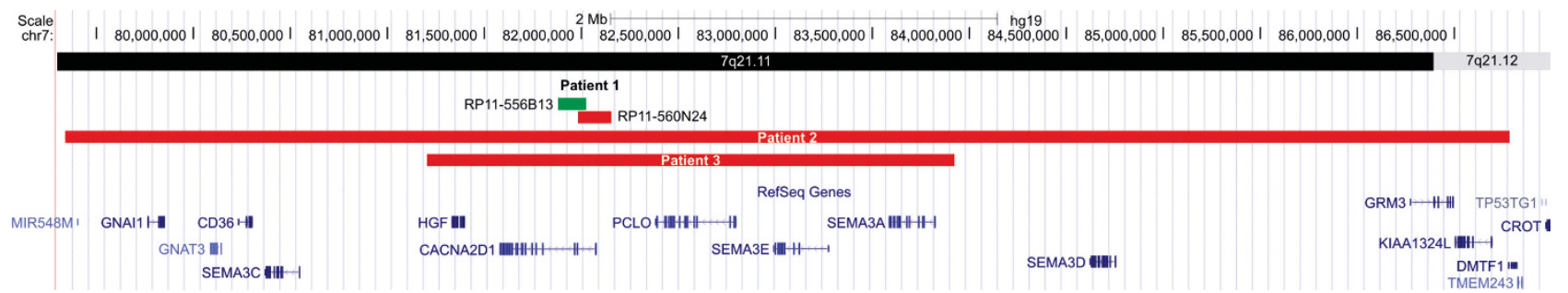

Figure 2 Overview of the aberrations on chromosome 7 in patients $1-3$. For patient 1, the flanking FISH probes are given. For patients 2 and 3 , the deleted region is shown. Genomic positions are according to GRCh37. 
phenotypes. The deletion in patient 3 was inherited from the mother who presents with mild ID but no epilepsy. The maternal grandfather also presented with mild ID and a maternal aunt with ID and epilepsy. Unfortunately, both could not be assessed for further testing. The aberration in patient 1 and 2 occurred de novo.

The breakpoint on the $\mathrm{X}$-chromosome in patient 1 resides in the centromeric region. The ARHGEF9 gene, of which deletion has been described in patients with epilepsy and ID, ${ }^{13-15}$ is located close to the breakpoint. To evaluate whether this gene might contribute to the more severe phenotype observed in patient 1 , both $\mathrm{X}$-inactivation as well as expression of ARHGEF9 were evaluated for this patient. ARHGEF9 is still expressed in this patient (Supplementary Figure 1) and total X-skewing was observed in favor of the abnormal $\mathrm{X}$-chromosome.

Although in our control cohort of approximately 5000 cases not one aberration in the CACNA2D1 gene could be detected, the database of genomic variants (http://projects.tcag.ca/variation/) contains three studies ${ }^{16-18}$ describing deletions comprising coding regions of CACNA2D1 in healthy individuals.

All of this suggests a broader spectrum of clinical phenotypes observed with CACNA2D1 aberrations.

Epilepsy was treated in patients 2 and 3 with valproic acid, which causes an increased concentration of gamma-aminobutyric acid in central nervous system synapses and blocks voltage-gated sodium channels and T-type calcium channels. ${ }^{19}$

Malformations of cortical development, including polymicrogyria, are an important cause of therapy-resistant epilepsy. ${ }^{20,21}$ In patient 1 , epilepsy is associated with polymicrogyria, which might explain the therapy resistant nature of the observed epilepsy in this patient.

Recently, mutations in the CACNA2D2 gene, which encodes another peptide of the $\alpha_{2} \delta$ subunit, have been associated with epilepsy. ${ }^{22,23}$ Mutations in the other isoforms have been linked to disorders such as Brugada syndrome, short QT syndrome (CACNA2D1), autosomal recessive cone dystrophy and night blindness (CACNA2D3) and autism spectrum disorders $(C A C N A 2 D 4){ }^{24-28}$ Deletion of the CACNA2D4 gene has been described in patients with bipolar disorder. ${ }^{29}$ Cardiac defects linked to Brugada syndrome and short QT syndrome associated with loss-of-function of CACNA2D1 were not observed in the patients reported here.

Besides epilepsy, all three patients also present with ID. Although haploinsufficiency of the CACNA2D1 gene could account for this, in patients 2 and 3, other genes in the deletion interval are likely to contribute to the observed phenotype. The PCLO gene and a group of semaphorin genes are located in the common deleted interval in patients 2 and 3 (Figure 2). PCLO encodes the piccolo presynaptic cytomatrix protein, which is involved in monoaminergic neurotransmission $^{30}$ and synaptic vesicle exocytosis ${ }^{31}$ and has already been associated with major depression disorder. ${ }^{32}$ The semaphorins have an important role in the development of the nervous system and in axonal guidance. ${ }^{33}$ A missense mutation in the SEMA3E gene has been described in a single patient with CHARGE Syndrome. ${ }^{34}$ The typical features associated with CHARGE Syndrome such as coloboma of the eye, heart defects and deafness were, however, not observed in patients 2 and 3. In patient 2, deletion of the GRM3 gene encoding the metabotropic glutamate receptor 3 protein could also contribute to the ID. This protein is involved in learning, memory, anxiety and the perception of pain. ${ }^{35}$

The obesity with hyperinsulinism in patient 2 is very likely to be caused by deletion of the CD36 gene (Figure 2). This gene encodes a glycoprotein of the platelet surface and may function in the transport and/or as a regulator of fatty acid transport. ${ }^{36}$ In mice, deletion of the
CD36 gene was found to be responsible for insulin resistance, defective fatty acid metabolism and hypertriglyceridemia. ${ }^{37}$ In humans, deficiency in CD36 is associated with insulin resistance in the Japanese population. ${ }^{38}$

To conclude, we report three new patients with epilepsy and ID in whom the CACNA2D1 gene is disrupted or deleted and as such is a candidate gene for the epileptic phenotype. In addition, in patient 2 , the obesity with hyperinsulinism could be explained by the deletion of the $C D 36$ gene.

Comprehensive studies will, however, be needed to further unravel the genotype-phenotype correlation associated with aberrations of the CACNA2D1 gene.

\section{CONFLICT OF INTEREST}

The authors declare no conflict of interest.

\section{ACKNOWLEDGEMENTS}

We are indebted to the patients and their parents. We thank Willy Lissens for the X-inactivation assay and Lies Vantomme for expert technical assistance. Sarah Vergult was supported by a PhD fellowship of the Research Foundation-Flanders (FWO) and is now supported by a postdoctoral grant from the Special Research Fund (BOF) from Ghent University. This article presents research results of the Belgian program of Interuniversity Poles of attraction initiated by the Belgian State, Prime Minister's Office, Science Policy Programming (IUAP). This study makes use of data generated by the DECIPHER Consortium. A full list of centers who contributed to the generation of the data is available from http://decipher.sanger.ac.uk and via email from decipher@sanger.ac.uk. Funding for the project was provided by the Wellcome Trust.

1 Ferreira MA, O'Donovan MC, Meng YA et al: Collaborative genome-wide association analysis supports a role for ANK3 and CACNA1C in bipolar disorder. Nat Genet 2008; 40: $1056-1058$

2 Jouvenceau A, Eunson LH, Spauschus A et al: Human epilepsy associated with dysfunction of the brain P/Q-type calcium channel. Lancet 2001; 358: 801-807.

3 Bhat S, Dao DT, Terrillion CE et al: CACNA1C (Ca(v)1.2) in the pathophysiology of psychiatric disease. Progr Neurobiol 2012; 99: 1-14.

4 Dolphin AC: Calcium channel auxiliary alpha2delta and beta subunits: trafficking and one step beyond. Nat Rev Neurosci 2012; 13: 542-555.

5 Thorpe AJ, Offord J: The alpha2-delta protein: an auxiliary subunit of voltagedependent calcium channels as a recognized drug target. Curr Opin Invest Drugs 2010; 11: 761-770.

6 Bian F, Li Z, Offord J et al: Calcium channel alpha2-delta type 1 subunit is the major binding protein for pregabalin in neocortex, hippocampus, amygdala, and spinal cord: an ex vivo autoradiographic study in alpha2-delta type 1 genetically modified mice. Brain Res 2006; 1075: 68-80.

7 Cole RL, Lechner SM, Williams ME et al: Differential distribution of voltage-gated calcium channel alpha-2 delta (alpha2delta) subunit mRNA-containing cells in the rat central nervous system and the dorsal root ganglia. J Comp Neurol 2005; 491: 246-269.

8 De Weer A, Poppe B, Vergult S et al: Identification of two critically deleted regions within chromosome segment 7q35-q36 in EVI1 deregulated myeloid leukemia cell lines. PLoS One 2010; 5: e8676.

9 Vergult S, Dauber A, Delle Chiaie B et al: 17q24.2 microdeletions: a new syndromal entity with intellectual disability, truncal obesity, mood swings and hallucinations. Eur J Hum Genet 2012; 20: 534-539.

10 Menten B, Pattyn F, De Preter K et al: ArrayCGHbase: an analysis platform for comparative genomic hybridization microarrays. BMC Bioinformatics 2005; 6: 124 .

11 Allen RC, Zoghbi HY, Moseley AB, Rosenblatt HM, Belmont JW: Methylation of Hpall and $\mathrm{Hhal}$ sites near the polymorphic CAG repeat in the human androgen-receptor gene correlates with X chromosome inactivation. Am J Hum Genet 1992; 51: 1229-1239.

12 Mefford HC, Yendle SC, Hsu C et al: Rare copy number variants are an important cause of epileptic encephalopathies. Ann Neurol 2011; 70: 974-985.

13 Marco EJ, Abidi FE, Bristow J et al: ARHGEF9 disruption in a female patient is associated with $\mathrm{X}$ linked mental retardation and sensory hyperarousal. J Med Genet 2008; 45: 100-105.

14 Shimojima K, Sugawara M, Shichiji M et al: Loss-of-function mutation of collybistin is responsible for X-linked mental retardation associated with epilepsy. J Hum Genet 2011; 56: 561-565.

15 Lesca G, Till M, Labalme A et al: De novo Xq11.11 microdeletion including ARHGEF9 in a boy with mental retardation, epilepsy, macrosomia, and dysmorphic features. Am J Med Genet A 2011; 155A: 1706-1711. 
16 Wang K, Li M, Hadley D et al: PennCNV: an integrated hidden Markov model designed for high-resolution copy number variation detection in whole-genome SNP genotyping data. Genome Res 2007; 17: 1665-1674.

17 Itsara A, Cooper GM, Baker C et al: Population analysis of large copy number variants and hotspots of human genetic disease. Am J Hum Genet 2009; 84: 148-161.

$18 \mathrm{Xu} \mathrm{H}$, Poh WT, Sim X et al: SgD-CNV, a database for common and rare copy number variants in three Asian populations. Hum Mutat 2011; 32: 1341-1349.

19 Chapman A, Keane PE, Meldrum BS, Simiand J, Vernieres JC: Mechanism of anticonvulsant action of valproate. Progr Neurobiol 1982; 19: 315-359.

20 Guerrini R, Dobyns WB, Barkovich AJ: Abnormal development of the human cerebral cortex: genetics, functional consequences and treatment options. Trends Neurosci 2008; 31: 154-162.

21 Leventer RJ, Guerrini R, Dobyns WB: Malformations of cortical development and epilepsy. Dialogues Clin Neurosci 2008; 10: 47-62.

22 Edvardson S, Oz S, Abulhijaa FA et al: Early infantile epileptic encephalopathy associated with a high voltage gated calcium channelopathy. J Med Genet 2013; 50 118-123.

23 Pippucci T, Parmeggiani A, Palombo F et al: A novel null homozygous mutation confirms CACNA2D2 as a gene mutated in epileptic encephalopathy. PLoS One 2013 8: e82154

24 Burashnikov E, Pfeiffer R, Barajas-Martinez $\mathrm{H}$ et al: Mutations in the cardiac L-type calcium channel associated with inherited J-wave syndromes and sudden cardiac death. Heart Rhythm 2010; 7: 1872-1882.

25 Templin C, Ghadri JR, Rougier JS et al: Identification of a novel loss-of-function calcium channel gene mutation in short QT syndrome (SQTS6). Eur Heart J 2011; 32: 1077-1088.

26 Iossifov I, Ronemus M, Levy D et al: De novo gene disruptions in children on the autistic spectrum. Neuron 2012; 74: 285-299.

27 Wycisk KA, Zeitz C, Feil S et al: Mutation in the auxiliary calcium-channel subunit CACNA2D4 causes autosomal recessive cone dystrophy. Am J Hum Genet 2006; 79 973-977.
28 Wycisk KA, Budde B, Feil S et al: Structural and functional abnormalities of retinal ribbon synapses due to Cacna2d4 mutation. Invest Ophthalmol Visual Sci 2006; 47 3523-3530.

29 Van Den Bossche MJ, Strazisar M, De Bruyne S et al: Identification of a CACNA2D4 deletion in late onset bipolar disorder patients and implications for the involvement of voltage-dependent calcium channels in psychiatric disorders. Am J Med Genet B Neuropsychiatric Genet 2012; 159B: 465-475.

30 Cen X, Nitta A, Ibi D et al: Identification of Piccolo as a regulator of behavioral plasticity and dopamine transporter internalization. Mol Psychiatry 2008; 13: 349 451-363.

31 Leal-Ortiz S, Waites CL, Terry-Lorenzo R, Zamorano P, Gundelfinger ED, Garner CC: Piccolo modulation of Synapsin 1a dynamics regulates synaptic vesicle exocytosis. J Cell Biol 2008; 181: 831-846.

32 Sullivan PF, de Geus EJ, Willemsen G et al: Genome-wide association for major depressive disorder: a possible role for the presynaptic protein piccolo. Mol Psychiatry 2009; 14: 359-375.

33 Yazdani U, Terman JR. The semaphorins. Genome Biol 2006; 7: 211.

34 Lalani SR, Safiullah AM, Molinari LM, Fernbach SD, Martin DM, Belmont JW: SEMA3E mutation in a patient with CHARGE syndrome. J Med Genet 2004; 41: e94.

35 Kandaswamy R, McQuillin A, Sharp SI et al: Genetic association, mutation screening, and functional analysis of a Kozak sequence variant in the metabotropic glutamate receptor 3 gene in bipolar disorder. JAMA Psychiatry 2013; 70 : 591-598.

36 Su X, Abumrad NA: Cellular fatty acid uptake: a pathway under construction. Trends Endocrinol Metab 2009; 20: 72-77.

37 Aitman TJ, Pravenec M, Kurtz TW, Scott J: The spontaneously hypertensive rat: model to dissect cellular defects in glucose and fatty acid metabolism. PathologieBiologie 1998; 46: 693-694.

38 Miyaoka K, Kuwasako T, Hirano K, Nozaki S, Yamashita S, Matsuzawa Y: CD36 deficiency associated with insulin resistance. Lancet 2001; 357: 686-687.

Supplementary Information accompanies this paper on European Journal of Human Genetics website (http://www.nature.com/ejhg) 